\title{
Blood eosinophils in COPD and the future risk of pneumonia
}

\author{
Pierre Ernst
}

Affiliation: McGill University, Lady Davis Research Institute, Jewish General Hospital, Montreal, QC, Canada.

Correspondence: Pierre Ernst, Centre for Clinical Epidemiology, Lady Davis Research Institute, Jewish General Hospital, 3755 Cote Ste-Catherine, Montreal, Quebec H3T 1E2, Canada. E-mail: pierre.ernstamcgill.ca

@ERSpublications

There is an increase in pneumonia risk in COPD patients who have higher blood eosinophil counts at baseline http://ow.ly/XPVh30kvOpZ

Cite this article as: Ernst P. Blood eosinophils in COPD and the future risk of pneumonia. Eur Respir J 2018; 52: 1800981 [https://doi.org/10.1183/13993003.00981-2018].

The eosinophil, whether in the blood or sputum, has been receiving increasing attention as a measure of treatment response or failure in patients with chronic obstructive pulmonary disease (COPD). A recent review by BAFADHEL et al. [1] summarises the biology of the eosinophil, its role in airway disease, as well as the clinical data supporting the use of blood eosinophils as a biomarker in airway disease, and more specifically, COPD. To paraphrase their conclusions: while the role of eosinophils in COPD needs clarification, there is now the realisation that blood eosinophils may be a useful guide to therapy. There is, however, substantial within-person variation in blood eosinophil counts [2]. Despite this, there is a significant proportion of patients with COPD in whom blood eosinophil counts are consistently high or low: $65 \%$ consistently above or below $0.40 \times 10^{9}$ per L over a 1 -year period in the Leicester cohort [1] and $51 \%$ at the $2 \%$ cut-off over a 3 -year period in the ECLIPSE study [3]. Consistency also appears better when using absolute counts and at lower counts. For example, using a cut-off value of $<0.15 \times 10^{9}$ per $\mathrm{L}$, Southworth et al. [4] found $87 \%$ to be in the same category at 6 months and a similar proportion at $>2$ years of follow-up. It has further been suggested that higher blood eosinophil counts are predictive of future eosinophilic exacerbations [5] and this is supported by the better response to oral corticosteroids in patients with exacerbations characterised by higher blood eosinophils at presentation [1]. The broader importance of the eosinophil in the natural history of airway disease at an early stage is suggested by the predictive value of blood eosinophil counts on decline in forced expiratory volume in $1 \mathrm{~s}(\mathrm{FEV} 1)$ and FEV1/forced vital capacity in young adults from the New Zealand birth cohort, an effect that is independent of asthma and cigarette smoking [6].

In the May issue of the European Respiratory Journal, VeDEL-KROGH et al. [7] described the relationship between blood eosinophil count at levels of $\geqslant 0.34 \times 10^{9}$ per $\mathrm{L}$ and risk of severe pneumonia requiring hospitalisation from the Copenhagen General Population Study, a prospective cohort study with spirometry and complete blood counts obtained at recruitment. There were 7180 individuals with airway obstruction by spirometry and without self-reported asthma, of whom 4832 had smoked $>10$ pack-years.

Overall, among these 4832 subjects, there was no relationship between blood eosinophil count and pneumonia. When stratifying individuals according to $\mathrm{FEV}_{1}$, among subjects with an $\mathrm{FEV}_{1} \geqslant 50 \%$ predicted, there was a trend toward a protective effect of higher eosinophils (incidence rate ratio (IRR) $0.69,95 \%$ CI $0.47-1.00)$. In subjects with severe airway obstruction and $\geqslant 10$ pack-years of smoking (553 subjects), the risk of pneumonia was increased (IRR 1.82, 95\% CI 1.10-3.02) after adjustment for various confounders including prior pneumonia events, use of inhaled corticosteroids (ICS) and number of 
inflammatory markers. When not counting multiple pneumonias in the same individual or when excluding those with a pneumonia event prior to cohort entry, the risk was further diminished and no longer statistically significant. The effect was stronger in a population with "clinical COPD" $(\geqslant 10$ pack-years of smoking, an exacerbation in the prior year and FEV $1<70 \%$ predicted; IRR 3.14, 95\% CI 1.42-6.91). However, we are not provided with an analysis not counting multiple pneumonias in the same individual or when excluding those with pneumonia prior to cohort entry, which to me are the most valid analyses.

The results of this study appear to contradict a patient level meta-analysis of clinical trials reported by PAVORD et al. [8]. Among 3065 subjects who were fairly typical of COPD clinical trial populations, although $41 \%$ were without prior exacerbation in previous 12 months, and who did not receive ICS in their treatment arm, the hazard ratio of pneumonia was 1.53 (95\% CI 1.01-2.31) for subjects with peripheral blood eosinophil counts at baseline of $<2 \%$ as compared to those with higher values. While the differences in results may reflect differences in study populations (one carried out in the general population, the other in a highly selected clinical trial population; differences in the definition of pneumonia; and the Copenhagen study examined only severe pneumonia leading to hospitalisation, the clinical trial looking at all pneumonia events), the results are still hard to reconcile. One has to wonder whether severe exacerbations of COPD might have been mislabelled as pneumonia in a general population cohort, especially since higher eosinophil counts were also a risk factor for severe COPD exacerbations leading to hospitalisation in the same subgroups of patients [9]. This might have been less likely to happen in clinical trials where exacerbations of COPD are a principal outcome. Of note, other retrospective re-analyses of clinical trials did not find differences in risk of pneumonia according to blood eosinophil count [10, 11].

From the point of view of biological plausibility, eosinopenia is the more obvious choice as a risk factor for pneumonia. Eosinophils appear to have potent antibacterial properties in a mouse model [12]. Major basic protein and eosinophilic cationic protein contained in the cytoplasmic granules of eosinophils are bactericidal [13]. In a prospective clinical cohort of fairly typical COPD patients, $32 \%$ of whom were current smokers and $86 \%$ receiving ICS, high bacterial load of potentially pathogenic organisms at baseline was inversely related to sputum eosinophils (but not blood eosinophils), while at the onset of a COPD exacerbation, blood eosinophils had decreased significantly in those with increased bacterial load [14].

Pneumonia is a common complication of COPD. Advancing age, current smoking, severity of airway obstruction, low body weight, prior pneumonia, recent exacerbations, use of high-dose ICS [15], especially fluticasone [16], are well recognised and important risk factors [17-21]. The additional predictive value of blood eosinophils, whether high or low, is uncertain. While the relationship between blood eosinophils and response to therapy in COPD is changing clinical practice, it is less clear how the relationship of eosinophils to pneumonia risk might change our approach. For the moment, it appears prudent to target ICS therapy to patients with COPD who have higher blood eosinophil counts since they derive the most benefit in order to balance the increase in risk of pneumonia which appears to be present at all levels of blood eosinophils [22].

Conflict of interest: None declared.

\section{References}

1 Bafadhel M, Pavord ID, Russell REK. Eosinophils in COPD: just another biomarker? Lancet Respir Med 2017; 5: 747-759.

2 Gibson PG. Variability of blood eosinophils as a biomarker in asthma and COPD. Respirology 2018; 23: 12-13.

3 Singh D, Kolsum U, Brightling CE, et al. Eosinophilic inflammation in COPD: prevalence and clinical characteristics. Eur Respir J 2014; 44: 1697-1700.

4 Southworth T, Beech G, Foden P, et al. The reproducibility of COPD blood eosinophil counts. Eur Respir J 2018; 52: 1800427.

5 Greulich T, Vogelmeier CF. Blood eosinophils as a marker of eosinophilic exacerbations in COPD. Lancet Respir Med 2018; 6: el7.

6 Hancox RJ, Pavord ID, Sears MR. Associations between blood eosinophils and decline in lung function among adults with and without asthma. Eur Respir J 2018; 51: 1702536.

7 Vedel-Krogh S, Nordestgaard BG, Lange P, et al. Blood eosinophil count and risk of pneumonia hospitalisations in individuals with COPD. Eur Respir J 2018; 51: 1800120.

8 Pavord ID, Lettis S, Anzueto A, et al. Blood eosinophil count and pneumonia risk in patients with chronic obstructive pulmonary disease: a patient-level meta-analysis. Lancet Respir Med 2016; 4: 731-741.

9 Vedel-Krogh S, Nielsen SF, Lange P, et al. Blood eosinophils and exacerbations in chronic obstructive pulmonary disease. The Copenhagen General Population Study. Am J Respir Crit Care Med 2016; 193: 965-974.

10 Pascoe S, Locantore N, Dransfield MT, et al. Blood eosinophil counts, exacerbations, and response to the addition of inhaled fluticasone furoate to vilanterol in patients with chronic obstructive pulmonary disease: a secondary analysis of data from two parallel randomised controlled trials. Lancet Respir Med 2015; 3: 435-442.

11 Siddiqui SH, Guasconi A, Vestbo J, et al. Blood eosinophils: a biomarker of response to extrafine beclomethasone/ formoterol in chronic obstructive pulmonary disease. Am J Respir Crit Care Med 2015; 192: 523-525. 
12 Linch SN, Kelly AM, Danielson ET, et al. Mouse eosinophils possess potent antibacterial properties in vivo. Infect Immun 2009; 77: 4976-4982.

13 Lehrer RI, Szklarek D, Barton A, et al. Antibacterial properties of eosinophil major basic protein and eosinophil cationic protein. J Immunol 1989; 142: 4428-4434.

14 Kolsum U, Donaldson GC, Singh R, et al. Blood and sputum eosinophils in COPD; relationship with bacterial load. Respir Res 2017; 18: 88.

15 Ernst P, Gonzalez AV, Brassard P, et al. Inhaled corticosteroid use in chronic obstructive pulmonary disease and the risk of hospitalization for pneumonia. Am J Respir Crit Care Med 2007; 176: 162-166.

16 Suissa S, Patenaude V, Lapi F, et al. Inhaled corticosteroids in COPD and the risk of serious pneumonia. Thorax 2013; 68: 1029-1036.

17 Farr BM, Bartlett CL, Wadsworth J, et al. Risk factors for community-acquired pneumonia diagnosed upon hospital admission. British Thoracic Society Pneumonia Study Group. Respir Med 2000; 94: 954-963.

18 Almirall J, Bolibar I, Balanzo X, et al. Risk factors for community-acquired pneumonia in adults: a population-based case-control study. Eur Respir J 1999; 13: 349-355.

19 Lange P, Vestbo J, Nyboe J. Risk factors for death and hospitalization from pneumonia. A prospective study of a general population. Eur Respir J 1995; 8: 1694-1698.

20 Crim C, Calverley PM, Anderson JA, et al. Pneumonia risk in COPD patients receiving inhaled corticosteroids alone or in combination: TORCH study results. Eur Respir J 2009; 34: 641-647.

21 Mullerova H, Chigbo C, Hagan GW, et al. The natural history of community-acquired pneumonia in COPD patients: a population database analysis. Respir Med 2012; 106: 1124-1133.

22 Suissa S, Ernst P. Precision Medicine Urgency: The case of inhaled corticosteroids in COPD. Chest 2017; 152: $227-231$. 